\title{
Postural Adjustments in Catching: On the Interplay Between Segment Stabilization and Equilibrium Control
}

\author{
Pieter Tijtgat, Jos Vanrenterghem, Simon J. Bennett, \\ Dirk De Clercq, Geert J.P. Savelsbergh, and Matthieu Lenoir
}

The purpose of this study was to investigate postural adjustments in one-handed ball catching. Specifically, the functional role of anticipatory postural adjustments (APA) during the initial arm raising and subsequent postural adjustments (SPA) for equilibrium control and ball-hand impact were scrutinized. Full-body kinematics and kinetics allowed an analysis of the mechanical consequences of raising up the arm and preparing for ball-hand impact. APA for catching were suggested to be for segment stabilization. SPA had a functional role for equilibrium control by an inverted pendulum mechanism but were also involved in preparing for the impact of the ball on the hand, which was illustrated by an increased postural response at the end of the movement. These results were compared with raising up the arm in a well-studied reaction-time task, for which an additional counter rotation equilibrium mechanism was observed. Together, our findings demonstrate that postural adjustments should be investigated in relation to their specific functional task constraints, rather than generalizing the functional role of these postural adjustments over different tasks.

Keywords: postural adjustments; catching; arm raising; segment stabilization; equilibrium control

Catching an approaching object while standing (e.g., cricket fielding, handball) demands postural control related to raising the arm upwards and toward the object, as well as the subsequent postural disequilibrium caused by the expected and actual mechanical impulse of the ball acting on the hand. However, despite there being extensive study of how the human perceptual-motor system is organized during catching (Zago, McIntyre, Senot, \& Lacquaniti, 2009), less attention

\footnotetext{
Tijtgat, De Clercq and Lenoir are with the Faculty of Medicine and Health Sciences, Department of Movement and Sports Sciences, Ghent University, Gent, Belgium. Vanrenterghem and Bennett are with the Faculty of Science, Research Institute for Sport and Exercise Sciences, Liverpool John Moores University, Byrom Street Campus, Liverpool, UK. Savelsbergh is with the Faculty of Human Movement Sciences, Research Institute MOVE, VU University Amsterdam, Amsterdam, The Netherlands, and also the Institute for Biomedical Research into Human Movement and Health, Manchester Metropolitan University, Manchester, UK.
} 
has been paid to the role that postural control might play during such actions. At a behavioral level, the relevance of this omission is evident in the finding that postural control can be decisive for successful catching performance. For example, performance in poor catchers was found to approach the level of good catchers when sitting or with the aid of a support (Davids, Bennett, Kingsbury, Jolley, \& Brain, 2000; Angelakopoulos, Davids, Bennett, Tsorbatzoudis, \& Grouios, 2005; Savelsbergh, Bennett, Angelakopoulos, \& Davids, 2005). Further, in the somewhat less disequilibrating task of catching a falling object while standing, it has been shown that before moving the relevant effectors for prime arm movement, there is an anticipatory muscle contraction in muscles that are responsible for postural control (Shiratori \& Latash, 2001; Li \& Aruin, 2007, 2009). To date, however, while it is understood that postural adjustments associated with prime arm movement (i.e., vertical raising) are attributable to different control mechanisms (see below for more detail), a comprehensive conceptual understanding of such mechanisms during the interception of an approaching object has yet to be reported.

The existence of anticipatory postural adjustments (APA) has been observed in a task relatively similar to catching an approaching object, where the participant raises the arm vertically from beside the hip to the horizontal forward position. When raising the arm while standing, body posture is disturbed as the moving arm causes a quick change of the body geometry, which leads to a forward acceleration of the segment center of mass (COM). The internal forces caused by this acceleration of the prime mover in turn result in destabilizing reaction forces on the supporting segments (Massion, 1992). To counteract the destabilizing consequences of these forces, the central nervous system is suggested to exhibit APA (Bouisset \& Zattara, 1987; Zattara \& Bouisset, 1988), which is believed to occur simultaneously with, or just before, the initiation of the voluntary movement by means of feedforward control (Cordo \& Nashner, 1982). For raising one or both arms as a reaction to an auditory or visual stimulus, the mechanical expression of APA has been reported as a backward shift of the center of pressure (COP) starting before movement onset (Aruin \& Latash, 1995; Hodges, Cresswell, \& Thorstensson, 1999; Cuisinier, Olivier, \& Nougier, 2005; Bleuse et al., 2006; Bleuse et al., 2008).

While early postural responses have most frequently been associated with an inverted pendulum acting at the level of the ankle, which generates anticipatory forward acceleration of the whole body COM to compensate for the ensuing inertial forces from prime mover motion, a comparison of model-based and experimental data has led to the suggestion that the initial postural motion of whole body COM is in fact passive and hence that APA are better explained as a side effect of local segment stabilization (Pozzo, Ouamer, \& Gentil, 2001; Patla, Ishac, \& Winter, 2002). Segment stabilization is needed to counter reactive torques from the arm acting on the shoulder, which in turn affect the hip, knee and ankle joints. This means that after the initial movement impulse and its disequilibrating effects, subsequent postural adjustments (SPA) are required to maintain whole body postural equilibrium. In addition to the inverted pendulum mechanism acting at the ankle, a potential counter-rotation mechanism exists, which acts primarily at hip-level and is often required to retain balance in more destabilizing situations (Hof, 2007). This mechanism involves increased horizontal ground reaction forces (GRF), which generate linear accelerations of the whole body COM, and is thus distinct but complementary to the inverted pendulum mechanism and the resulting 
torque generation at the ankle. Indeed, the counter-rotation mechanism rotates the GRF vector, whereas the inverted pendulum mechanism translates the GRF vector.

Putting aside recent debate on the functional role of observations identified as APA (Pozzo et al., 2001; Patla et al., 2002), their presence and subsequent SPA have been well described in reactive arm raising tasks. This has provided a useful baseline for understanding postural adjustments in arm raising tasks, but as alluded to above there are important differences compared with one-handed catching, which could influence the mechanisms involved in maintaining postural balance. Raising the arm in reaction to an external stimulus demands a relatively simple and internally-timed intralimb coordination, whereas one-handed catching requires a precise spatiotemporal coordination between the catching arm and approaching ball to bring the catching hand to the right place at the right time (Tijtgat, Bennett, Savelsbergh, De Clercq, \& Lenoir, 2010). Therefore, in addition to countering disequilibrium due to raising the arm, one-handed catching often involves a reversal of the forward displacement of the arm to reduce impact at ball-hand contact to controllable levels (Williams \& McCririe, 1988). This can be expected to influence SPA, with the latter task potentially involving the two complementary equilibrium control mechanisms (i.e., inverted pendulum and counter rotation). Here, then, we report a study that was designed to comprehensively evaluate postural adjustments in one-handed catching, and thereby the contribution of APA and SPA, using mechanical observations.

\section{Method}

\section{Participants}

Six male self-declared right handed participants (aged between 21 and 23, mean weight $74.9 \pm 7 \mathrm{~kg}$ and mean height $180.3 \pm 4 \mathrm{~cm}$ ) gave their written informed consent (approved by the Ethical Committee of the host University) before participating.

\section{Materials and Procedure}

Participants were asked to stand still with their feet parallel (two marked lines) on a force plate (AMTI, $1000 \mathrm{~Hz}$ ), arms besides the body and head upright with the gaze directed forward. The experiment consisted of 10 catching trials $(\mathrm{CATCH})$. Participants were instructed to catch yellow, midpressured tennis balls. At the completion of the movement, the end position was retained for more than $3 \mathrm{~s}$ so that a new equilibrium-position was established. Balls were launched at a speed of $15.8 \pm 0.16 \mathrm{~m} / \mathrm{s}$ at $8.4 \mathrm{~m}$ from the participant's frontal plane by a ball-projection machine (Promatch/Mubo B.V., Gorinchem, The Netherlands). The machine was covered with black plastic that had a small cut-out section through which the balls were released so that participants could not anticipate ball delivery. Launching angle was adjusted so that balls arrived above participant's right shoulder. Spatial standard deviation of the interception point was $4.40 \mathrm{~cm}$ in the medio-lateral and $7.61 \mathrm{~cm}$ in the vertical direction (reflective of the variability of the ball machine); and $7.86 \mathrm{~cm}$ in the anterior-posterior direction as a result of the interaction between ball trajectory and forward movement of the hand. After catching, participants performed a reaction-time action (RAISE). They were instructed to raise their right arm as 
fast as possible until horizontal from the moment they saw a ball coming out of a ball machine. For RAISE, launching angle was changed so that while balls were still delivered toward the participant, they could not reach the participants' body. Participants wore headphones that minimized sound generated by the ball machine and a face shield to protect the face, while not disturbing access to the full visual field. Three-dimensional kinematic data were collected at $200 \mathrm{~Hz}$ using 12 infrared cameras (Proreflex, Qualisys AB, Gothenburg, Sweden). Markers were bilaterally placed on distal phalanx of hallux and digitus minimus, lateral and medial aspect of calcaneus, malleolus and femoral condyles, iliac crest, anterior and posterior superior iliac spine, sternum, C7, intertubercular sulcus of humerus, styloid process of radius and ulna, distal phalanx of thumb and index.

\section{Data Processing}

Kinetic and kinematic data were recorded simultaneously and filtered (low-pass Butterworth filter at $20 \mathrm{~Hz}$ for kinetics and at $10 \mathrm{~Hz}$ for kinematics). A 14-segment model consisting of feet, shanks, thighs, pelvis, thorax/abdomen, upper arms, lower arms and hands was developed using Visual 3D (C-Motion Inc., Gaithersburg, MD, USA).

Flexion and extension angles were calculated for elbow, shoulder, hip and ankle and expressed relative to their baseline level (between 500 and $300 \mathrm{~ms}$ before movement onset). COP anterior-posterior displacement was also expressed relative to the average position between 500 and $300 \mathrm{~ms}$ before movement onset. Onsets were calculated according to the criterion that the acceleration of the signal had to exceed $0.5 \mathrm{~m} / \mathrm{s}^{2}$ for at least $25 \mathrm{~ms}$. Onset of arm movement $\left(\mathrm{t}_{0}\right)$ was derived from the forward acceleration of the wrist. Onset of postural adjustments was derived from the anterior or posterior acceleration of COP. Movement time (MT) was the time between $\mathrm{t}_{0}$ and the end of the deceleration phase $\left(\mathrm{t}_{\mathrm{end}}\right)$.

The effect of raising the arm was quantified for both tasks by calculating COM-displacement and changes in momentum of key segments of the moving body. COM and momentum of the whole body (body), the right arm (consisting of right upper arm, lower arm and hand) and of the body without the right arm (rest of body), were derived. Finally, flexion and extension angles of hip and ankle together with resultant anterior-posterior COP-displacement and ground reaction forces (GRF) were included to enable a conclusive analysis of the equilibrium control mechanisms as suggested by Hof (2007).

\section{Statistical Analysis}

Intraparticipant mean data from 10 successful trials for CATCH and RAISE were calculated. Due to the small sample size, statistical tests consisted of nonparametric Wilcoxon tests. The level of significance was set at $p<.05$.

\section{Results}

\section{Arm Movement}

The catching movement $(\mathrm{CATCH})$ was recognized by substantial elbow flexion $(45 \pm 4 \mathrm{deg})$ at $\mathrm{t}_{0}$ that preceded shoulder flexion (Figure 1). As a consequence, the 

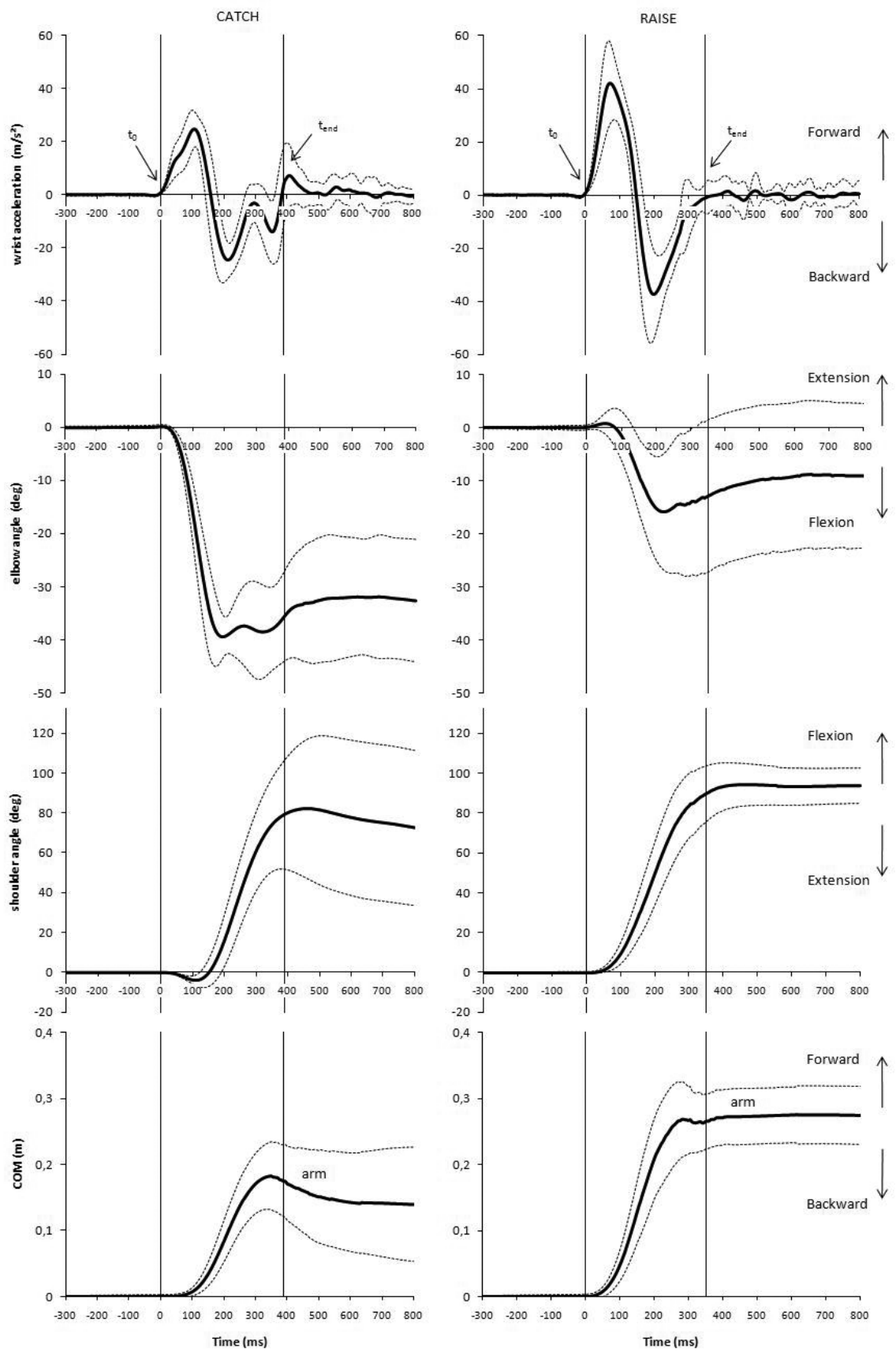

Figure 1 - Acceleration of wrist, angular displacement of elbow and shoulder, and displacement of COM of arm for CATCH (left panel) and RAISE (right panel). Group mean data and its standard deviation (dashed lines) are presented. First vertical line is $\mathrm{t}_{0}$, second vertical line is $t_{\text {end }}$. 
forward displacement of the COM of the arm was $10 \mathrm{~cm}$ less forward as compared with RAISE $(z=-2.207, p<.05)$, notwithstanding a comparable accelerationdeceleration profile of the wrist, though with more prominent peak amplitudes in $\operatorname{RAISE}(z=-2.207, p<.05)$.

\section{APA}

With respect to the mechanical observation of APA, no clear backward shift in COP before $t_{0}$ was observed. Onset of COP-shift was reached on average at $23 \pm 45 \mathrm{~ms}$ $(\mathrm{CATCH})$ and $3.33 \pm 48 \mathrm{~ms}$ before $\mathrm{t}_{0}$ (RAISE), and was not significantly different between conditions $(z=-1.363, n s)$.

Raising the arm for catching resulted in a similar forward displacement of arm $\mathrm{COM}$ and forward change in arm momentum (Figure 2). This was compensated by a backward displacement of COM of the rest of the body, and an opposing momentum of the rest of the body that closely matched the arm movement. The net result of the interplay between the displacements of arm and rest of body was a forward displacement of the whole body COM (Figure 2).

\section{SPA}

Shortly before ball-hand contact, the arm was moved backward in anticipation of ball impact, resulting in a backward change of momentum of the arm (Figure 2). This was preceded by a forward change in momentum of the rest of the body that was larger for CATCH than for RAISE $(z=-2.207, p<.05)$. The net result of this interplay between postural control for arm raising and grasping was a sustained forward momentum of the body.

An initial forward GRF was followed by a slight hip flexion and a backward GRF (Figure 3). The hip was extended in the second part of the catching phase and led to increased forward GRF. At the ankle joint, a small plantar flexion in the ankle and a forward shift of COP was observed, followed by dorsiflexion and COP that returned backward (Figure 4). As compared with CATCH, the RAISE task was characterized by a clear dynamic hip flexion and extension movement. As a consequence, the initial posterior GRF changed rapidly to a forward GRF, followed again by a backward GRF (Figure 3). Plantar flexion in the ankle was also observed, together with a COP that was initially shifted forward, albeit with a backward return followed by forward shift near the end of movement (Figure 4).

\section{Discussion}

The present study evaluated in one-handed catching the presence of APA with its role for segment stabilization or primary equilibrium control, and SPA with its role for equilibrium control and countering ball impact effects. One-handed catching did not exhibit consistent APA involving backward COP shifts before arm movement onset. Early postural adaptations supported the notion of segmental stabilization more than equilibrium control. The observed SPA leading up to ball contact supported both proposed equilibrium control mechanisms, involving postural modification associated to ball impact. We will consider each of these aspects in 


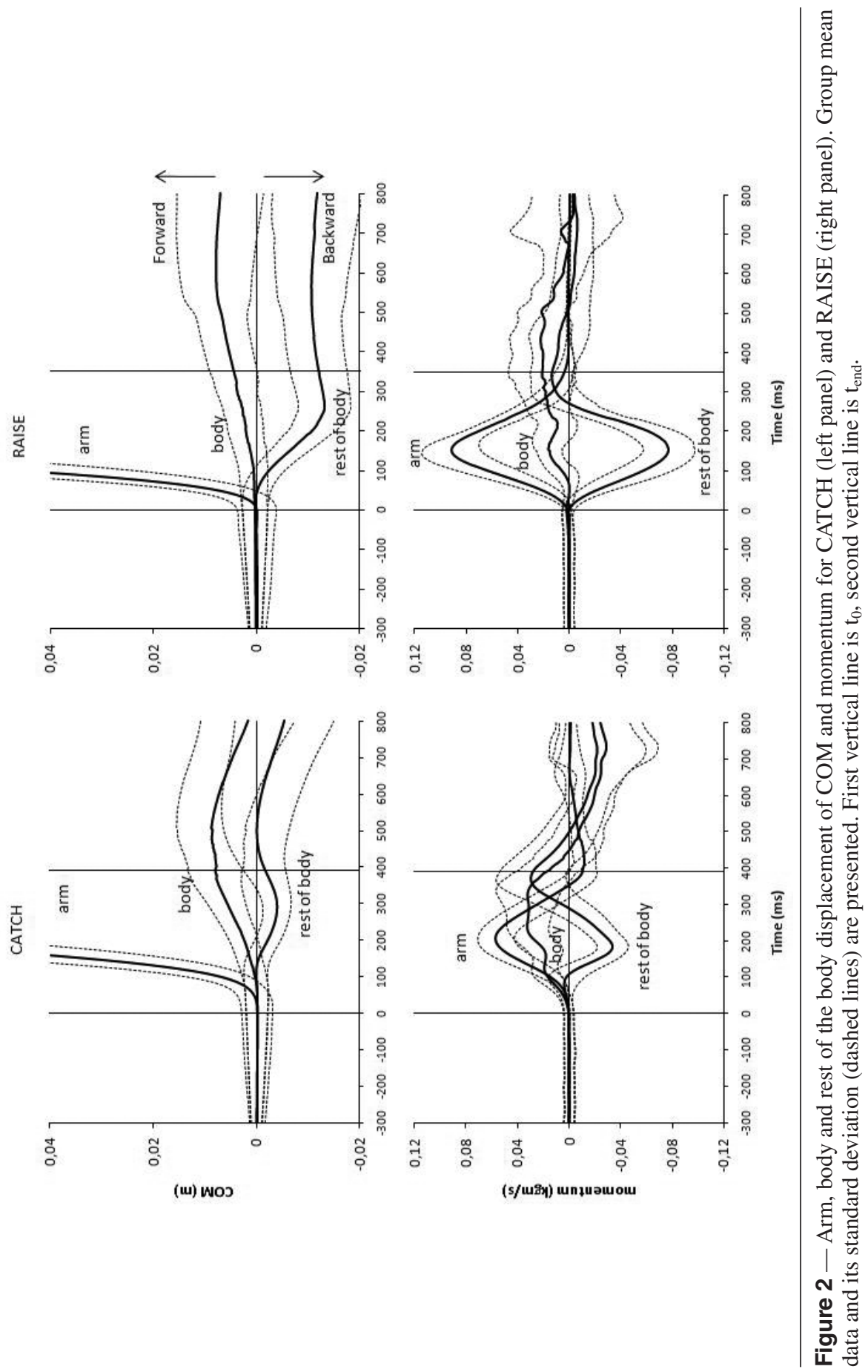


CATCH
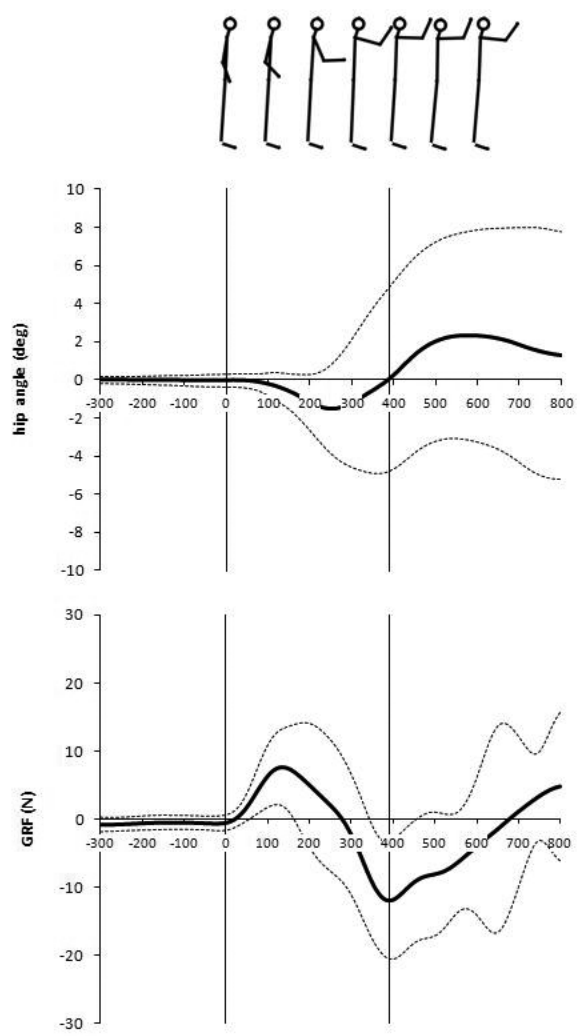

RAISE
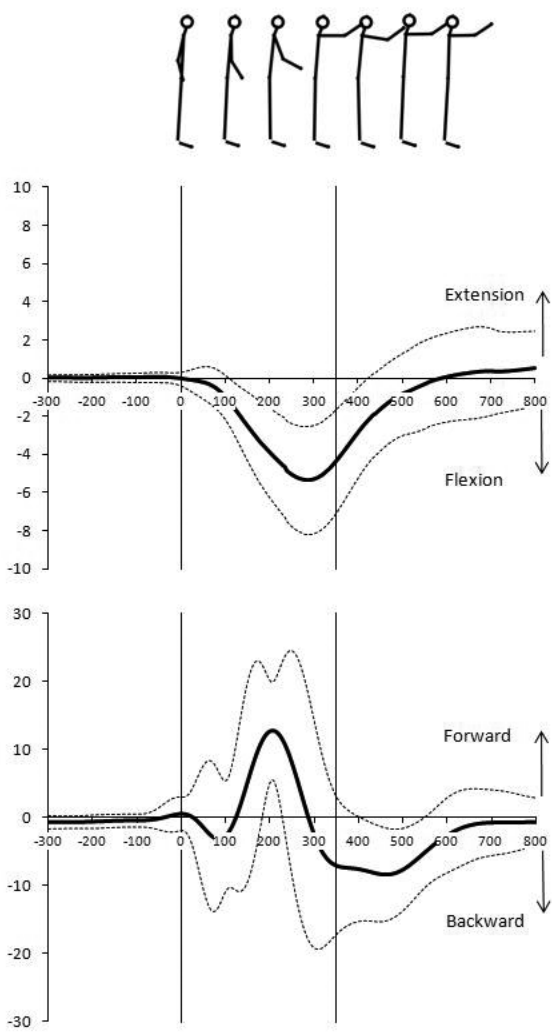

Figure 3 - Counter-rotating mechanism. Stick-diagram of participant DM. Group mean data and standard deviation (dashed lines) of the angular displacement of hip and displacement of GRF for CATCH (left panel) and RAISE (right panel). First vertical line is $\mathrm{t}_{0}$, second vertical line is $t_{\text {end }}$.

the light of previous studies, and where relevant provide supportive or countering argumentation from the comparison with reactive arm raising.

\section{APA Before Movement Onset}

The presence of APA has typically been evidenced by a consistent backward shift of COP-displacement before $\mathrm{t}_{0}$ (Aruin \& Latash, 1995; Hodges et al., 1999; Cuisinier et al., 2005; Hirschfeld, 2007; Girolami et al., 2010; Aimola et al., 2011), and has been represented by mean values from single representative subjects. In this experiment, however, no representative subject could be selected. On the contrary, our results showed large intra- and intersubject variability in COP-displacement at $\mathrm{t}_{0}$ (see Figure 5), with backward and forward shifts, as well as no shifts. Other studies have also failed to show a consistent backward movement of the COP before movement onset (De Wolf, Slijper, \& Latash, 1998; Nougier, Teasdale, Bard, \& 
$\mathrm{CATCH}$
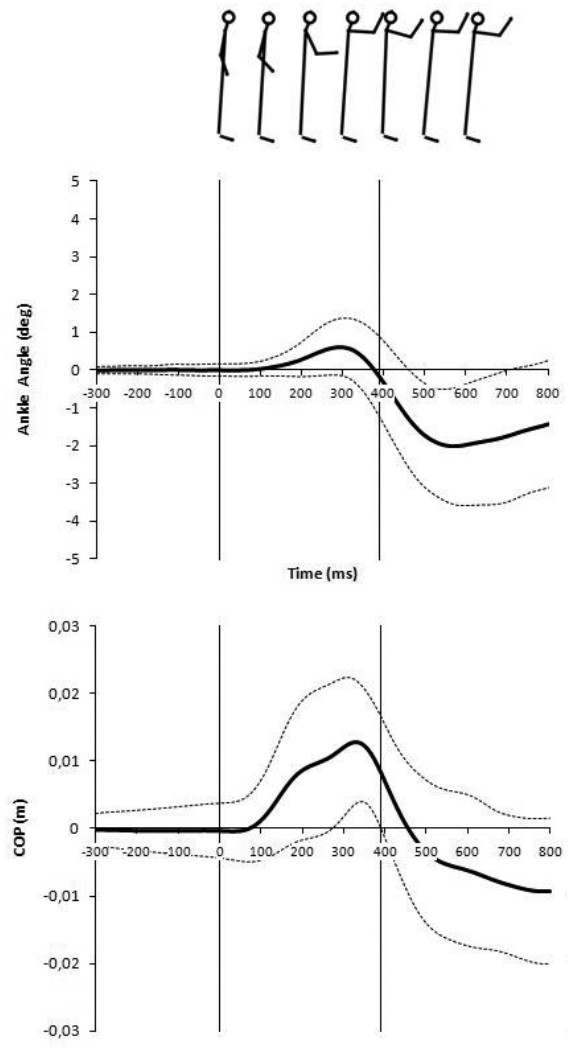

RAISE
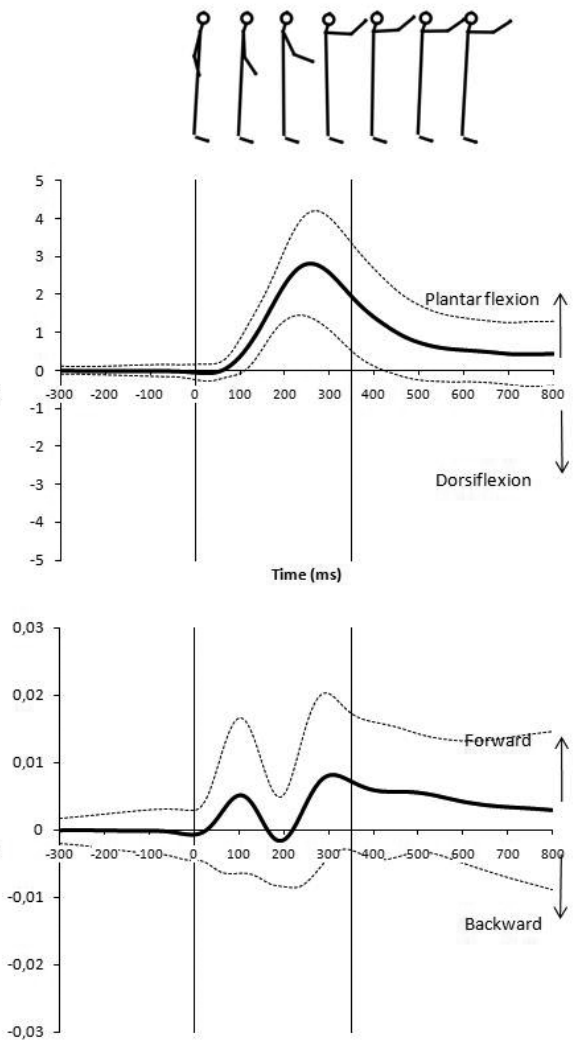

Figure 4 - Inverted pendulum mechanism. Stick-diagram of participant DM (rotation at the hip segment is abandoned). Group mean data and standard deviation (dashed lines) of the angular displacement of ankle and displacement of COP for CATCH (left panel) and RAISE (right panel). First vertical line is $t_{0}$, second vertical line is $t_{\text {end }}$.

Fleury, 1999; Hay \& Redon, 2001; Ferry, Martin, Termoz, Cote, \& Prince, 2004; Mochizuki, Ivanova, \& Garland, 2004). While such variability has rarely been reported in literature on APA in arm raising, it is notable that there is some suggestion from work on catching a falling ball with an outstretched arm that "signals obtained from force plate showed large variability across subjects" (Shiratori \& Latash, 2001, p. 1251). A potential explanation for the lack of finding APA clearly before movement onset in catching may be the presence of elbow flexion observed, since raising the arm when the elbow is flexed involves less destabilization (van der Fits, Klip, van Eykern, \& Hadders-Algra, 1998; Bleuse et al., 2006; Bleuse et al., 2008). Nevertheless, also in the RAISE task, where considerably less elbow flexion took place than during catching, no consistent APA before movement onset was observed (Figure 5, right panel), which seems in conflict with some suggestions made in the literature. Taken together, our findings indicate that the 

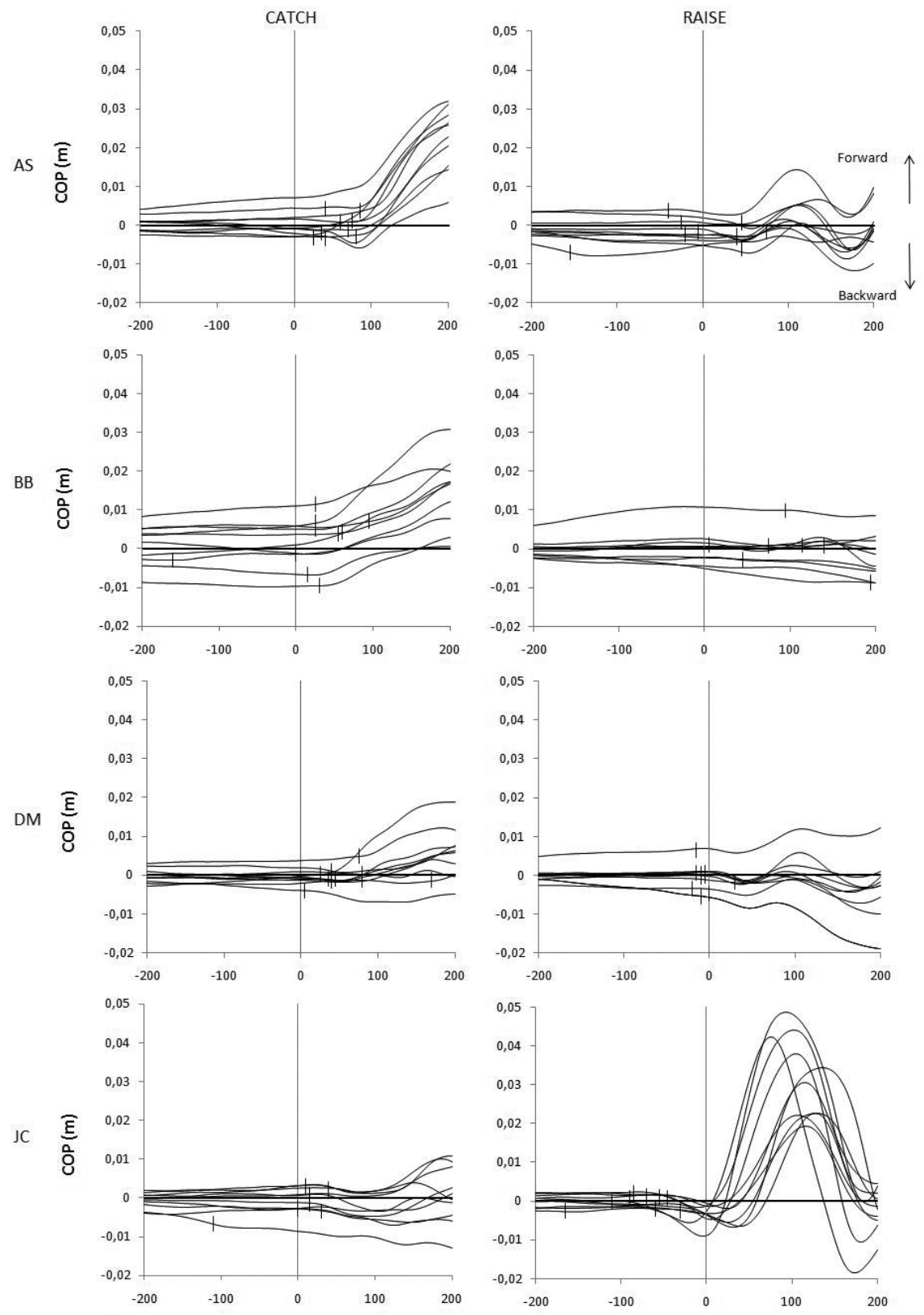

(continued)

Figure 5 - COP-displacement traces for CATCH (left panel) and RAISE (right panel) around $\mathrm{t}_{0}$. All trials for all subjects are represented. Onset of COP is indicated with ticks. Vertical line is $t_{0}$. 

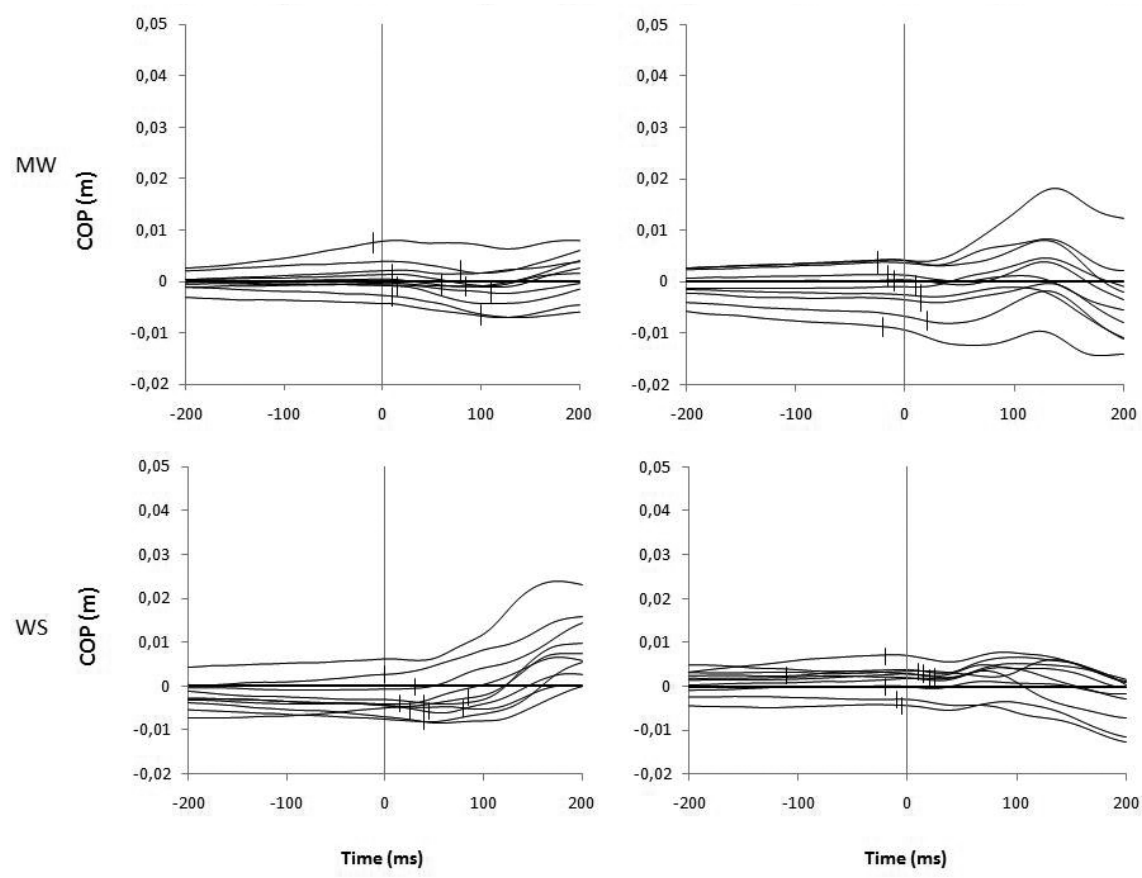

Figure 5 - continued

proposed backward shift of COP before movement onset is not a ubiquitous indicator of APA, and that if APA is considered present its functional role for whole body equilibrium control should be questioned (Pozzo et al., 2001; Patla et al., 2002). The initial motion of whole body COM might be a passive consequence of reaction forces due to counter-rotating segments rather than an active control of postural equilibrium, with antagonistic coactivations for segment stabilization. The generation of whole body forward momentum was up to $0.04 \mathrm{~kg} . \mathrm{m} / \mathrm{s}$. Considering that less forward momentum was observed in RAISE, strengthens the argument that successful catching and supportive segment stabilization is prioritized over equilibrium control during APA.

\section{SPA for Equilibrium Control}

After initial segment stabilization, an inverted pendulum strategy at the level of the ankle was found to be the predominant postural strategy for the catching movement (Hof, 2007), with angular displacement at the ankle explaining the COP-shifts. This supports the notion that active equilibrium control of the whole body COM occurs only $200 \mathrm{~ms}$ after $\mathrm{t}_{0}$ (Patla et al., 2002). Due to segment stabilization, the hip joint was only marginally flexed, followed by a hip extension and continued forward momentum of the rest of the body. This would be counter intuitive in the light of equilibrium control, but seems indicative of a postural control mechanism to anticipate the ensuing impact of the ball at the hand. In comparison, for RAISE a larger 
and dynamic hip flexion-extension coupling suggests an additional counter-rotating mechanism at hip level in addition to the inverted pendulum mechanism (Hof, 2007). Despite larger changes in momentum of the arm, the forward momentum of the rest of the body at $t_{\text {end }}$, was $34 \%$ less than in CATCH where a ball impact was evident around this time. Clearly, these differences in postural control between both tasks indicate that postural adjustments to voluntary movements are closely related to specific task constraints (see also Ilmane \& LaRue, 2008). Future work could reveal how postural adjustments might differ between different spatial or temporal constraints during interceptive actions.

Finally, some limitations of the current study have to be acknowledged. This study was limited to a sagittal plane analysis only considering the anterior-posterior direction of movement. Accordingly, axial rotations that could influence equilibrium control during unilateral arm raising were not analyzed (Bouisset \& Zattara, 1987). In addition, the observed findings only allow to suggest possible postural control strategies. A future in depth analysis through forward dynamic simulations could establish the true causative nature between reaction forces due to the prime mover and concomitant postural adjustments.

\section{Conclusion}

During arm raising for unconstrained catching, initial postural adjustments by feedforward control seem to be a consequence of the inertia of the movement itself (segment stabilization), rather than a mechanism to immediately counter disequilibrium. Afterward, an inverted pendulum mechanism accommodates equilibrium control when raising the arm for catching, whereas increased postural control seems to be warranted in preparation of ball impact on the hand. When raising the arm in a simple reaction-time task, SPA by an inverted pendulum was observed but with an additional counter-rotating mechanism to maintain balance. Overall, our findings demonstrated that the spatio-temporal constraints of one-handed catching lead to postural adjustments specific for that task, notwithstanding that both $\mathrm{CATCH}$ and RAISE disturb body posture in a similar way by raising the arm.

\section{References}

Aimola, E., Santello, M., La Grua, G., \& Casabona, A. (2011). Anticipatory postural adjustments in reach-to-grasp: effect of object mass predictability. Neuroscience Letters, 15 , 84-88. PubMed doi:10.1016/j.neulet.2011.07.027

Angelakopoulos, G.T., Davids, K., Bennett, S.J., Tsorbatzoudis, H., \& Grouios, G. (2005). Postural stability and hand preference as constraints on one-handed catching performance in children. Journal of Motor Behavior, 37, 377-385. PubMed doi:10.3200/ JMBR.37.5.377-385

Aruin, A.S., \& Latash, M.L. (1995). Directional specificity of postural muscles in feedforward postural reactions during fast voluntary arm movements. Experimental Brain Research, 103, 323-332. doi:10.1007/BF00231718

Bleuse, S., Cassim, F., Blatt, J.L., Labyt, E., Bourriez, J.L., Derambure, P., . . .. (2008). Anticipatory postural adjustments associated with arm movement in Parkinson's disease: a biomechanical analysis. Journal of Neurology, Neurosurgery, and Psychiatry, 79, 881-887. PubMed doi:10.1136/jnnp.2006.107318 
Bleuse, S., Cassim, F., Blatt, J.L., Labyt, E., Derambure, P., Guieu, J.D., . . .. (2006). Effect of age on anticipatory postural adjustments in unilateral arm movement. Gait \& Posture, 24, 203-210. PubMed doi:10.1016/j.gaitpost.2005.09.001

Bouisset, S., \& Zattara, M. (1987). Biomechanical study of the programming of anticipatory postural adjustments associated with voluntary movement. Journal of Biomechanics, 20, 735-742. PubMed doi:10.1016/0021-9290(87)90052-2

Cordo, P.J., \& Nashner, L.M. (1982). Properties of postural adjustments associated with rapid arm movements. Journal of Neurophysiology, 47, 287-382. PubMed

Cuisinier, R., Olivier, I., \& Nougier, V. (2005). Effects of foreperiod duration on anticipatory postural adjustments: determination of an optimal preparation in standing and sitting for a raising arm movement. Brain Research Bulletin, 66, 163-170. PubMed doi:10.1016/j.brainresbull.2005.04.010

Davids, K., Bennett, S., Kingsbury, D., Jolley, L., \& Brain, T. (2000). Effects of postural constraints on children's catching behavior. Research Quarterly for Exercise and Sport, 71, 69-73. PubMed

De Wolf, S., Slijper, H., \& Latash, M.L. (1998). Anticipatory postural adjustments during self-paced and reaction-time movements. Experimental Brain Research, 121, 7-19. PubMed doi:10.1007/s002210050431

Ferry, M., Martin, L., Termoz, N., Cote, J., \& Prince, F. (2004). Balance control during an arm raising movement in bipedal stance: which biomechanical factor is controlled? Biological Cybernetics, 91, 104-114. PubMed doi:10.1007/s00422-004-0501-7

Girolami, G.L., Shiratori, T., \& Aruin, A.S. (2010). Anticipatory postural adjustments in children with typical motor development. Experimental Brain Research, 205, 153-165. PubMed doi:10.1007/s00221-010-2347-7

Hay, L., \& Redon, C. (2001). Development of postural adaptation to arm raising. Experimental Brain Research, 139, 224-232. PubMed doi:10.1007/s002210100752

Hirschfeld, H. (2007). Motor control of every day motor tasks: guidance for neurological rehabilitation. Physiology \& Behavior, 92, 161-166. PubMed doi:10.1016/j.physbeh.2007.05.018

Hodges, P., Cresswell, A., \& Thorstensson, A. (1999). Preparatory trunk motion accompanies rapid upper limb movement. Experimental Brain Research, 124, 69-79. PubMed doi:10.1007/s002210050601

Hof, L. (2007). The equations of motion for a standing human reveal three mechanisms for balance. Journal of Biomechanics, 40, 451-457. PubMed doi:10.1016/j.jbiomech.2005.12.016

Ilmane, N., \& LaRue, J. (2008). Modulation of anticipatory postural adjustments in a complex task under different temporal constraints. Motor Control, 12, 330-347. PubMed

Li, X., \& Aruin, A. (2009). The effect of short-term changes in body mass distribution on feed-forward postural control. Journal of Electromyography and Kinesiology, 19, 931-941. PubMed doi:10.1016/j.jelekin.2008.05.003

Li, X., \& Aruin, A.S. (2007). The effect of short-term changes in the body mass on anticipatory postural adjustments. Experimental Brain Research, 181, 333-346. PubMed doi:10.1007/s00221-007-0931-2

Massion, J. (1992). Movement, posture and equilibrium: interaction and coordination. Progress in Neurobiology, 38, 35-56. PubMed doi:10.1016/0301-0082(92)90034-C

Mochizuki, G., Ivanova, T.D., \& Garland, S.J. (2004). Postural muscle activity during bilateral and unilateral arm movements at different speeds. Experimental Brain Research, 155, 352-361. PubMed doi:10.1007/s00221-003-1732-x

Nougier, V., Teasdale, N., Bard, C., \& Fleury, R. (1999). Modulation of anticipatory postural adjustments in a reactive and a self-triggered mode in humans. Neuroscience Letters, 260, 109-112. PubMed doi:10.1016/S0304-3940(98)00961-6

Patla, A.E., Ishac, M.G., \& Winter, D.A. (2002). Anticipatory control of center of mass and joint stability during voluntary arm movement from a standing posture: interplay 
between active and passive control. Experimental Brain Research, 143, 318-327. PubMed doi:10.1007/s00221-001-0968-6

Pozzo, T., Ouamer, M., \& Gentil, C. (2001). Simulating mechanical consequences of voluntary movement upon whole-body equilibrium: the arm-raising paradigm revisited. Biological Cybernetics, 85, 39-49. PubMed doi:10.1007/PL00007995

Savelsbergh, G.J.P., Bennett, S.J., Angelakopoulos, G.T., \& Davids, K. (2005). Perceptualmotor organization of children's catching behaviour under different postural constraints. Neuroscience Letters, 373, 153-158. PubMed doi:10.1016/j.neulet.2004.10.011

Shiratori, T., \& Latash, M.L. (2001). Anticipatory postural adjustments during load catching by standing subjects. Clinical Neurophysiology, 112, 1250-1265. PubMed doi:10.1016/ S1388-2457(01)00553-3

Tijtgat, P., Bennett, S.J., Savelsbergh, G.J.P., De Clercq, D., \& Lenoir, M. (2010). Advance knowledge effects on kinematics of one-handed catching. Experimental Brain Research, 201, 875-884. PubMed doi:10.1007/s00221-009-2102-0

van der Fits, I.B.M., Klip, A.W.J., van Eykern, L.A., \& Hadders-Algra, M. (1998). Postural adjustments accompanying fast pointing movements in standing, sitting and lying adults. Experimental Brain Research, 120, 202-216. PubMed doi:10.1007/s002210050394

Williams, J.G., \& McCririe, N. (1988). Control of arm and fingers during ball catching. Journal of Human Movement Studies, 14, 241-247.

Zago, M., McIntyre, J., Senot, P., \& Lacquaniti, F. (2009). Visuo-motor coordination and internal models for object interception. Experimental Brain Research, 192, 571-604. PubMed doi:10.1007/s00221-008-1691-3

Zattara, M., \& Bouisset, S. (1988). Posturo-kinetic organization during the early phase of voluntary upper limb movement. 1. Normal subjects. Journal of Neurology, Neurosurgery, and Psychiatry, 51, 956-965. PubMed doi:10.1136/jnnp.51.7.956 